\title{
Article \\ Correlation between High PD-L1 and EMT/Invasive Genes Expression and Reduced Recurrence-Free Survival in Blood-Circulating Tumor Cells from Patients with Non-Muscle-Invasive Bladder Cancer
}

\author{
Maria Beatrice Morelli ${ }^{1, *}+{ }^{+} \mathbb{D}$, Consuelo Amantini ${ }^{2,+}$, Jacopo Adolfo Rossi de Vermandois ${ }^{3}$, \\ Marilena Gubbiotti ${ }^{4}(\mathbb{D})$, Antonella Giannantoni ${ }^{5,6}{ }^{(D)}$, Ettore Mearini ${ }^{3}$, Federica Maggi ${ }^{1,7} \mathbb{D}^{1}$, Massimo Nabissi $^{1}(\mathbb{D}$, \\ Oliviero Marinelli ${ }^{1}\left(\mathbb{D}\right.$, Matteo Santoni ${ }^{8}$, Alessia Cimadamore ${ }^{9}$, Rodolfo Montironi ${ }^{10}$ (D) and Giorgio Santoni ${ }^{1, *}$ (D)
}

1 School of Pharmacy, Section of Experimental Medicine, University of Camerino, 62032 Camerino, Italy; federica.maggi@uniroma1.it (F.M.); massimo.nabissi@unicam.it (M.N.); oliviero.marinelli@unicam.it (O.M.)

2 School of Biosciences and Veterinary Medicine, University of Camerino, 62032 Camerino, Italy; consuelo.amantini@unicam.it

3 Urologic and Andrologic Clinics, University of Perugia, 05100 Perugia, Italy; ja.rossidevermandois@aospterni.it (J.A.R.d.V.); ettore.mearini@unipg.it (E.M.)

check for updates

Citation: Morelli, M.B.; Amantini, C. Rossi de Vermandois, J.A.; Gubbiotti, M.; Giannantoni, A.; Mearini, E.; Maggi, F.; Nabissi, M.; Marinelli, O.; Santoni, M.; et al. Correlation between High PD-L1 and EMT/Invasive Genes Expression and Reduced Recurrence-Free Survival in Blood-Circulating Tumor Cells from Patients with Non-Muscle-Invasive Bladder Cancer. Cancers 2021, 13, 5989. https://doi.org/10.3390/ cancers13235989

Academic Editor: Michelle R. Downes

Received: 16 November 2021 Accepted: 24 November 2021 Published: 28 November 2021

Publisher's Note: MDPI stays neutral with regard to jurisdictional claims in published maps and institutional affiliations.

Copyright: (c) 2021 by the authors. Licensee MDPI, Basel, Switzerland. This article is an open access article distributed under the terms and conditions of the Creative Commons Attribution (CC BY) license (https:/ / creativecommons.org/licenses/by/ $4.0 /)$.
4 Department of Urology, San Donato Hospital, 52100 Arezzo, Italy; marilena.gubbiotti@uslsudest.toscana.it

5 Department of Medical and Surgical Sciences, University of Siena, 53100 Siena, Italy; antonella.giannantoni@unisi.it

6 Neurosciences, Functional and Surgical Urology Unit, Santa Maria alle Scotte Hospital, 53100 Siena, Italy

7 Department of Molecular Medicine, University Sapienza, 00185 Rome, Italy

8 Oncology Unit, Macerata Hospital, 62100 Macerata, Italy; mattymo@alice.it

9 Section of Pathological Anatomy, Department of Biomedical Sciences and Public Health School of Medicine, Polytechnic University of Marche Region, Umberto I Hospitals, 60121 Ancona, Italy; a.cimadamore@staff.univpm.it

10 Molecular Medicine and Cell Therapy Foundation, Department of Clinical and Molecular Sciences, Polytechnic University of Marche Region, 60126 Ancona, Italy; r.montironi@univpm.it

* Correspondence: mariabeatrice.morelli@unicam.it (M.B.M.); giorgio.santoni@unicam.it (G.S.); Tel.: +39-0737403312 (M.B.M.); +39-0737403319 (G.S.)

+ These authors contributed equally to this work.

Simple Summary: The correlation between immune checkpoint-programmed death-ligand 1 (PD-L1) marker and epithelial-mesenchymal transition (EMT) status may help to identify potential biomarkers for the use of immune checkpoint blockades and other immunotherapy approaches in non-muscle invasive bladder cancer (NMIBC) recurrent patients. The aim of our study was to assess to potential use of PD-L1, TWIST1, ZEB1, VIMENTIN and TIMP2 mRNA expression as prognostic biomarkers.

Abstract: Background: PD-L1 represents a crucial immune checkpoint molecule in the tumor microenvironment, identified as a key target for cancer immunotherapy. A correlation between $P D-L 1$ and EMT-related genes expression in various human cancers has been suggested. Methods: By ScreenCell filtration, digital droplet PCR and confocal microscopy analysis, we aimed to investigate the expression of PD-L1 and EMT/invasive genes (TWIST1, ZEB1, VIMENTIN, TIMP2) in circulating tumor cells (CTCs) collected from the blood of non-muscle-invasive bladder cancer (NMIBC) patients, assessing the prognostic value of these biomarkers in the disease. Welchs' test and Mann-Whitney $\mathrm{U}$ test, correlation index, Kaplan-Meier, Univariate and Multivariate Cox hazard proportional analysis were used. Results: Higher PD-L1, TIMP2 and VIM mRNA levels were found in pT1 compared to pTa NMIBC. As evaluated by Kaplan-Meier and Univariate and Multivariate Cox analysis, enhancement of PD-L1, TWIST1 and TIMP2 expression reduces the recurrent free survival in NMIBC patients. Conclusions: High PD-L1, TWIST1 and TIMP2 mRNAs mark the recurrent-NMIBC patients and by reducing the RFS represent negative prognostic biomarkers in these patients.

Keywords: NMIBC; CTC; immunotherapy; PD-L1; EMT; TWIST1; TIMP2; RFS 


\section{Introduction}

Over 400,000 patients are newly diagnosed with bladder cancer (BC) every year [1]. Three quarters of them have tumors that do not invade the detrusor muscle, which are described as non-muscle-invasive bladder cancers (NMIBCs) [2]. NMIBCs confined to the mucosa or invading the lamina propria are classified as stage Ta or T1, respectively, accordingly to the TNM classification system, whereas flat high-grade tumors confined to the mucosa are classified as CIS (Tis) [2]. NMIBCs are characterized by a relatively good prognosis, allowing most NMIBC patients to undergo only local treatment to prevent tumor recurrence and progression to muscle-invasive $\mathrm{BC}$ (MIBCs) that presents worse prognosis for metastatic disease. Treatment of NMIBC patients is based on the risk of tumor recurrence and/or progression [2]. Patients with high risk NMIBC are treated with transurethral resection of bladder tumor (TURBT) followed in some cases by Bacille Calmette Guerin (BCG) instillations. Standard treatment for patients with tumors that recur also after adequate BCG treatment is the surgical removal of the bladder (cystectomy), which is associated to high risk of complications, mortality and reduction of patient's quality life [3,4].

The immune checkpoint marker, programmed death-1 (PD-1), and its ligand, programmed death ligand-1 (PD-L1, CD274, B7-H1), have recently attracted interest in the field of cancer immunology [5]. PD-L1 is frequently overexpressed in tumors [5]. By binding to PD-1, it may be possible to inhibit the activation of T lymphocytes in order to evade the host immune response, preventing tumors from cytotoxic $\mathrm{T}$ lymphocyte-induced killing. PD-L1 also interacts with B7.1 to further suppress the tumor antigen-induced activation of cytotoxic T lymphocytes. The blockade of this pathway using specific inhibitors such as pembrolizumab, nivolumab or atezolizumab could enhance the cytotoxicity of $\mathrm{T}$ cells in the tumor environment and substantially increase long-term survival in different cancers [5,6].

$\mathrm{BCs}$ have been characterized as a tumor group in which the immunological response is well conserved. $P D-L 1$ is expressed in $B C$ and recent studies have suggested that it represents a mediator of stage progression [7], with $B C$ patients expressing high $P D-L 1$ levels showing a poor prognosis and relapse-free survival. As of January 2020, the FDA approved pembrolizumab (anti-PD-1) for treating high risk BCG unresponsive NMIBC patients [8]. This phase II trial reported a 3-month clinical complete response in $44.6 \%$ of Tis patients, $41.7 \%$ of $\mathrm{T} 1$ and concomitant CIS patients, with $52.6 \%$ of complete responses lasting over 12 months and no progression to MIBC [8]. Moreover, in a comparable phase II trial (SWOG S1605) evaluating the PD-L1 inhibitor atezolizumab, a 3-month complete response rate in $41.1 \%$ of patients with Ta/T1 tumors and concomitant CIS (NCT02844816) was reported [9].

Recent evidence in cancers suggests a relationship between $P D-L 1$ and epithelial mesenchymal transition (EMT) gene expression [10,11]. EMT is a multi-step process involving the transition from an epithelial to mesenchymal phenotype in response to chemoresistance and is important for cancer metastasis and recurrence [12-15]. EMT upregulates PD-L1 expression through the phosphoinositide 3-kinase/protein kinase B pathway in breast cancer [16]. Analyzing data from the Cancer Genome Atlas (TCGA) database, patients with positive $P D-L 1$ expression and EMT have a worse prognosis compared to those with positive $P D-L 1$ and negative EMT in head and neck squamous cell carcinoma [17]; moreover, a negative prognostic potential as well as high risk of recurrence and tumor progression have been reported in esophageal squamous cell carcinoma (ESCC) [11].

At present, biomarkers predictive of recurrence or progression for NMIBC are currently lacking. Liquid biopsy and, in particular, circulating tumor cells (CTCs) are currently under investigation to address this need $[18,19]$. CTCs are tumor cells that originate from a primary tumor, flowing through the bloodstream and circulating throughout the body, which may contribute to hematogenous metastasis [20]. Meta-analysis has shown that in MIBC patients CTCs are significantly associated with tumor progression and poor overall survival (OS) [21]. However, to date, blood CTCs have been understudied in NMIBC, although they are identified in these tumors. 
Given the potential of CTCs for risk stratification and the lack of information relative to prognostic factors in NMIBC patients, we isolated CTCs in the blood from NMIBC patients undergoing TURBT, to evaluate the expression of $P D-L 1$ and its correlation with EMT markers by digital droplet PCR (ddPCR). We also investigated the correlation of these markers with clinic-pathological features and time to recurrence to determine their prognostic significance.

\section{Materials and Methods}

\subsection{Patients and Ethic Statement}

Peripheral blood was collected before TURBT from 50 patients with a diagnosis of NMIBC (Tis, Ta and T1) undergoing TURBT from 2018 to 2020 at the Urologic and Andrologic Clinics, University of Perugia, Perugia, Italy. The patients were heterogeneous with respect to their clinical course. All the patients or their guardians provided written informed consent according to the research proposals approved by the Ethical Committee CEAS UMBRIA (Ethic Committee approval code: URO009-3171/18). The use of patient data and CTCs for research purposes at the University of Perugia has been executed pursuant to Italian legislation and international standards.

\subsection{CTC Blood Sample Processing}

Peripheral blood was collected into ScreenCell blood collection tubes. $7.5 \mathrm{~mL}$ of each blood sample collected in a K2-EDTA was processed for $3 \mathrm{~h}$ using ScreenCell devices (Sarcelles, France) according to the manufacturer's protocol. ScreenCell filters were washed with RPMI 1640 medium and Red Blood Lysis Buffer (MiltenyBiotec, Bologna, Italy). Then, enrichment filter-adherent CTCs were collected in RPMI medium and cell suspension filtered again. The ScreenCell microfiltration is an epitope-independent size-based device, able to capture all CTCs in the bloodstream. It has been used in CTC identification in rare tumors like hemangiopericytoma [22] as well as in more common tumors including bladder cancer [23].

\subsection{RNA Extraction}

Total RNA from isolated CTCs was extracted by using the Single Shot Cell Lysis Kit (BioRad, Hercules, CA, USA) according to the protocol.

\subsection{Reverse Transcription}

Total RNA was retrotranscribed by Iscript Advanced cDNA Synthesis kit (BioRad) and the resulting cDNA was used to preamplify each sample for all primers used in the gene expression analysis by SSOADvancedPreAmp Kit and PrimePCRPreAMP Assays (BioRad, Hercules, CA, USA).

\subsection{Digital Droplet PCR (ddPCR)}

The ddPCRSupermix for Probes (No dUTP) (BioRad) and the specific PrimePCR ${ }^{\mathrm{TM}}$ $\mathrm{ddPCR}^{\mathrm{TM}}$ Expression Probe Assays conjugated with FAM or HEX fluorescent dyes (the same pool used in the preamplification step) (BioRad) were then used to perform the ddPCR. The following target genes were analyzed: PD-L1, zinc finger E-box binding homebox 1 (ZEB1), Twist family BHLH transcription factor 1 (TWIST1), Vimentin (VIM) and the invasive gene TIMP Metallopeptidase Inhibitor 2 (TIMP2). Data, normalized to $\beta$-actin concentration, were analyzed using the QuantaSoft Software (BioRad). Since some of the analyzed transcripts could also be expressed, although at low levels, in normal blood cells ddPCR analysis was carried out identifying the CD3D, CD19, CD45, ICAM1, Beta2-microglobulin, ITGA2B/CD41, GYPA/CD235a gene expression in NMIBC samples, comparing the results with those obtained from peripheral blood mononuclear cells from a normal human donor and taking them as negative threshold.

\subsection{CTC Sample Staining}

To further confirm the ddPCR data, CTCs were fixed in $4 \%$ paraformaldehyde for 5 min at room temperature. Then, cells were permeabilized by using $0.3 \%$ Triton X-100 in PBS 
for $15 \mathrm{~min}$ at room temperature. Then, the blocking solution (3\% BSA, 0.3\% Triton X-100 in PBS) was added for $60 \mathrm{~min}$ at room temperature. Staining was performed using mouse anti-human pan-Cytokeratin (C11) Ab (PanCK; 1:50, sc-8018, Santa Cruz Biotechnology, Heidelberg, Germany), anti-human CD45 Ab (1:50, \#13917, Cell Signaling Technology, Danvers, MA, USA) and anti-human EpCAM (VU1D9) Ab (1:50, \#2929, Cell Signaling Technology) followed by goat anti-mouse IgG H\&L Ab (Alexa Fluor ${ }^{\circledR}$ 594, 1:100, Abcam, Cambridge, UK), labeled with PureBlu ${ }^{\mathrm{TM}}$ DAPI (\#1351303, BioRad) according to the manufacturer's protocol. Slides were then analyzed under 60X magnification with C2 Plus confocal laser scanning microscope (Nikon Instruments, Firenze, Italy). Optimized emission detection bandwidths were configured by Zeiss Zen control software. Images were processed using NIS Element Imaging Software (Nikon Instruments, Firenze, Italy). Candidate CTCs were identified from top ranked cells by one of two trained technicians. In accordance with the manufacturer's protocol, the definition for epithelial CTCs required cells to have a DAPI-positive nucleus with a diameter $\geq 4 \mu \mathrm{m}, \mathrm{CK}$ or EpCAM staining surrounding $\geq 50 \%$ of the nucleus, and the absence of staining in the counterstain channel (CD45).

\subsection{Statistical Analysis}

This was a pilot study. The analysis of frequency distribution was performed using a Chi-squared test selecting as expected frequencies: <60 for age; male category for sex; papillary for histology; pathological grade and stage. $p<0.05$ was considered as statistically significant. Recurrence-free survival (RFS) was defined as the time from TURBT to the clinical or histological evidence of tumor recurrence. We determined, by Relative Operating Characteristic (ROC) curve analysis, the expression value for each analyzed gene that best discriminated between recurrence or not. In addition, the Welch's $t$-test and MannWhitney $\mathrm{U}$ test were used to compare gene expression using GraphPad Prism version 7.00 for Windows, GraphPad Software (La Jolla, California, CA, USA, www.graphpad.com, accessed on 16 October 2021). $p<0.05$ was considered as statistically significant. KaplanMeier analysis was also used for RFS analysis. For Univariate analysis of significance, the long rank test or Multivariate Cox analysis MedCalc Statistical Software version 16.4.3 (MedCalc Software bv, Ostend, Belgium; https: / / www.medcalc.org, accessed on 16 October 2021) was used. $p<0.05$ was considered as statistically significant.

\subsection{Availability of Data}

Data is available upon request.

\section{Results}

\subsection{Detection of CTCs in the Blood of NMIBC Patients}

All 50 patients enrolled in this study had histologically confirmed diagnosis of NMIBC. The list of patients' characteristics, including average age, sex, grade, T stage classification, histology, infiltration, recurrence and therapy, is shown in Table 1.

CTCs were isolated from blood samples in patients undergoing TURBT. Among the 50 NMIBC patients, 2 patients were not considered since they underwent BCG or BCG plus Mitomycin $C$ instillations. Then, we found that among 48 NMIBC patients collected by ScreenCell filtration, 5 samples were CTCs negative, whereas 43/48 (89.6\%) had detectable CTCs, with a median CTC count of about 4.0 (range 2-52). The CTC phenotype was directly evaluated by ddPCR, by analyzing the EpCAM and CK expression in all 43 filtered samples (Table 2). Data, normalized to $\beta$-actin concentration, were evaluated using the QuantaSoft Software and expressed as ratio between cDNA copies/ $\mu \mathrm{L}$ of the variable and $\beta$-actin. Digital droplet PCR is highly sensitive (at level of single cells), highly specific, does not require personal interpretation, permits the identification of both EpCAM positive or negative CTCs and also simultaneously allows us to check the CTC purity, censoring the samples exceeding the cut-off for blood contaminant cells. 
Table 1. Patients' demographic and clinical characteristic and classification based on WHO 2017 criteria.

\begin{tabular}{cc}
\hline Data & Patients \\
\hline Gender, $\boldsymbol{n}$ (\%) & \\
Male & $41(82 \%)$ \\
Female & $9(18 \%)$ \\
Age years & $(47-93)$ \\
Range & 73.2 \\
Media & 74 \\
Median & $37(74 \%)$ \\
Tumor histology & $13(26 \%)$ \\
Papillary & \\
Not Papillary & $40(80 \%)$ \\
Pathological Tumor Grade & $10(20 \%)$ \\
Low & $1(2 \%)$ \\
High & $3(6 \%)$ \\
Pathological Tumor Stage & $36(72 \%)$ \\
Tx & $10(20 \%)$ \\
Tis & \\
Ta & $20(40 \%)$ \\
T1 & $30(60 \%)$ \\
Yecurrence & \\
No & $5(10 \%)$ \\
Tissue Infiltration & $45(90 \%)$ \\
Yes & $2(4 \%)$ \\
No & $48(96 \%)$ \\
Instillations &
\end{tabular}

Table 2. Gene expression evaluated by ddPCR. Bold: ID patients.

\begin{tabular}{cccccc}
\hline & EPCAM & CK & & EPCAM & CK \\
\hline NMIBC 1 & 0.2458 & 0.31145 & NMIBC 23 & 0.10238 & 0.06905 \\
\hline NMIBC 2 & 0.1179 & 0.16667 & NMIBC 24 & 0.34483 & 0.16034 \\
\hline NMIBC 3 & 0.4273 & 0.33752 & NMIBC 25 & 0.0416 & 0.00000 \\
\hline NMIBC 4 & 0.0315 & 0.36292 & NMIBC 26 & 0.0691 & 0.05647 \\
\hline NMIBC 5 & 0.2771 & 0.11547 & NMIBC 27 & 0.1963 & 0.12313 \\
\hline NMIBC 6 & 0.344 & 0.13298 & NMIBC 28 & 0.2425 & 0.14434 \\
\hline NMIBC 7 & 0.1339 & 0.0324 & NMIBC 29 & 0.0484 & 0.04449 \\
\hline NMIBC 8 & 0.4008 & 0.2776 & NMIBC 30 & 0.0516 & 0.01572 \\
\hline NMIBC 9 & 0.1803 & 0.03673 & NMIBC 31 & 0.07946 & 0.04948 \\
\hline NMIBC 10 & 0.0858 & 0.11489 & NMIBC 32 & 0.02271 & 0.15895 \\
\hline NMIBC 11 & 0.2591 & 0.22878 & NMIBC 33 & 0.41441 & 0.20751 \\
\hline NMIBC 12 & 0.50918 & 0.28367 & NMIBC 34 & 0.095 & 0.12333 \\
\hline NMIBC 13 & 0.05625 & 0.04 & NMIBC 35 & 0.22199 & 0.12474 \\
\hline NMIBC 14 & 0.6005 & 0.04381 & NMIBC 36 & 0.01373 & 0.00000 \\
\hline NMIBC 15 & 0.09119 & 0.20061 & NMIBC 37 & 0.17112 & 0.24293 \\
\hline NMIBC 16 & 0.02533 & 0.06267 & NMIBC 38 & 0.21712 & 0.08065 \\
\hline NMIBC 17 & 1.27187 & 0.11111 & NMIBC 39 & 0.07692 & 0.15858 \\
\hline NMIBC 18 & 0.1788 & 0.49721 & NMIBC 40 & 0.17618 & 0.10277 \\
\hline NMIBC 19 & 0.1521 & 0.07606 & NMIBC 41 & 0.20774 & 0.12194 \\
\hline NMIBC 20 & 0.3 & 0.18844 & NMIBC 42 & 0.37625 & 0.27421 \\
\hline NMIBC 21 & 0.02426 & 0.05391 & NMIBC 43 & 0.12639 & 0.00000 \\
\hline NMIBC 22 & 0.052 & 0.07067 & & & \\
\hline
\end{tabular}


Data, normalized to $\beta$-actin concentration, were evaluated using the QuantaSoft Software and expressed as ratio between cDNA concentration of gene target and $\beta$-actin Here we found that 40/43 (about 93\%) of the CTCs examined were EpCAM-positive and CK-positive; 3 of 43 (7.0\%) CTCs were EpCAM-positive but CK-negative and consequently were censored as non-conventional CTC (Table 3).

Table 3. Blood patients' CTCs.

\begin{tabular}{cc}
\hline Percentage CTCs Positive after Cell Screen Filtration & $\boldsymbol{n}(\mathbf{\%})$ \\
\hline Positive & $43(89.6 \%)$ \\
Negative & $5(10.4 \%)$ \\
\hline CTC specificity after ddPCR \\
\hline EpCAM + /CK + /CD45- \\
EpCAM + /CK - /CD45- \\
EpCAM - /CK + /CD45- & $40(93.0 \%)$ \\
Patients pathological grade and stage & $3(7.0 \%)$ \\
Total & $0(0.0 \%)$ \\
Tis & $\boldsymbol{n} \mathbf{( \% )}$ \\
Ta LG & $40(100 \%)$ \\
Ta HG & $3(7.5 \%)$ \\
T1 LG & $27(67.5 \%)$ \\
T1 HG & $4(10.0 \%)$ \\
\end{tabular}

The CTC phenotype was further evaluated by confocal microscopy for the expression of EpCAM, pan-CK, CD45 and DAPI (Figure 1).

EpCAM/DAPI

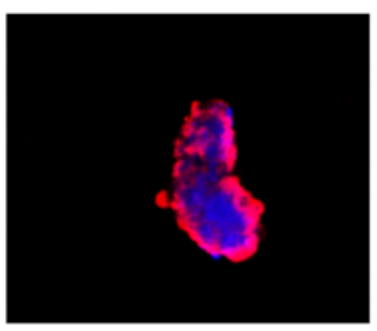

pan-CK/DAPI

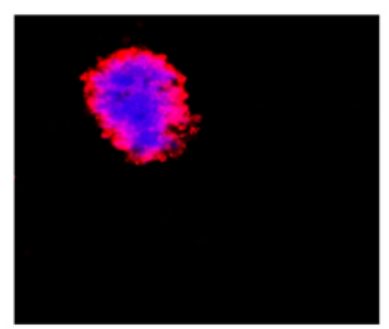

CD45/DAPI

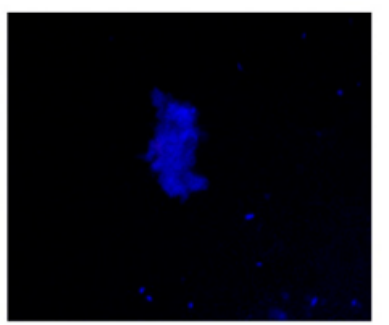

Figure 1. Confocal microscopy representative images of isolated CTCs stained for epithelial and hematopoietic markers. Representative image by confocal microscopy of circulating cancer cells stained with anti-human EPCAM, anti-human pan-CK or anti-human CD45 Abs followed by goat anti-mouse secondary antibody Alexa 594-conjugated. DAPI was used to counteract nuclei. Magnification $100 \times . \quad \mathrm{CK}=$ cytokeratin, an epithelial cytoplasmic marker; DAPI = nuclear marker; EpCAM = epithelial membrane marker; CD45 = leukocyte common antigen.

Recurrence is the major problem of NMIBC. EMT has been suggested to identify patients at high risk of developing a progressive disease in pTa [15] and the role of PD-L1 in $\mathrm{BC}$ recurrence has been suggested [2].

Herein, we found 14 cases of recurrence out of all 40 CTC-positive patients at the first follow up: two were Tis, nine were Ta and three were T1 (Figure 2A,B). At the second follow up, among 14 patients, 7 out of 10 experienced recurrence (Figure 2C). The proportion of Ta to Tis was six out of seven vs. one out of seven, respectively (Figure 2D). Six out of seven patients who were positive at the second follow-up were already positive at the first. 
A

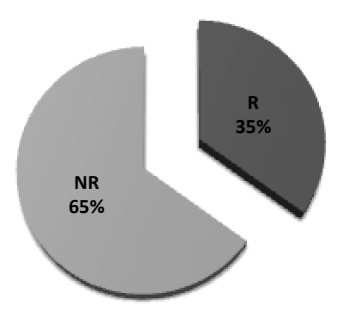

C

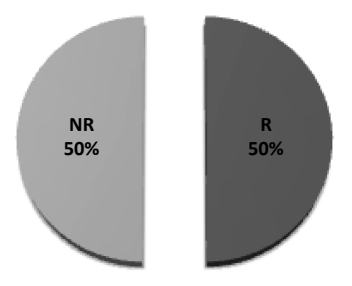

B

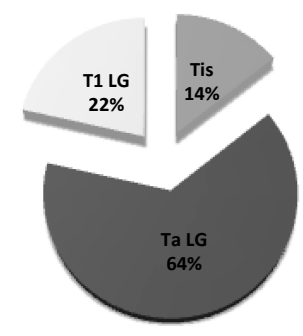

D

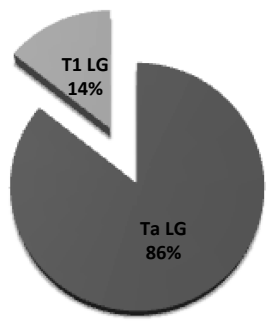

Figure 2. Pie charts of proportions for recurrence: (A) Patients' distribution at the first follow up; (B) T stage distribution in patients developing recurrence at the first follow up; (C) patients' distribution at the second follow up; (D) T stage distribution in patients developing recurrence at the second follow up. $\mathrm{R}=$ recurrent; $\mathrm{NR}=$ non recurrent.

\subsection{Detection of Different EMT Markers in CTCs from NMIBC Patients}

The expression of the EMT markers EpCAM and Vimentin (VIM) was evaluated in NMIBC patients by ddPCR and ROC analysis. We found that $35 \%$ and $45 \%$ of NMIBC samples showed low EpCAM or VIM expression, respectively (Table 4).

Table 4. Correlation between EpCAM or VIM expression levels in blood CTCs and pathological stage $\mathrm{T}$ in NMIBC patients.

\begin{tabular}{cc}
\hline EpCAM $^{\text {low }}$ & $(\boldsymbol{n = 1 4 / 4 0 , 3 5 . 0 \% )}$ \\
\hline Tis & $1 / 14(7.1 \%)$ \\
Ta & $9 / 14(64.3 \%)$ \\
T1 & $4 / 14(28.6 \%)$ \\
\hline EpCAM & $(\boldsymbol{n}=\mathbf{2 6 / 4 0 , 6 5 . 0} \%)$ \\
\hline Tis & $2 / 26(7.7 \%)$ \\
Ta & $22 / 26(84.6 \%)$ \\
T1 & $2 / 26(7.7 \%)$ \\
\hline VIM & $(\boldsymbol{n}=\mathbf{1 8} / \mathbf{4 0}, \mathbf{4 5 . 0} \%)$ \\
\hline Tis & $3 / 18(16.7 \%)$ \\
Ta & $13 / 18(72.2 \%)$ \\
T1 & $2 / 18(11.1 \%)$ \\
\hline VIM & $(\boldsymbol{n}=\mathbf{2 2} / \mathbf{4 0}, \mathbf{5 5 . 0} \%)$ \\
\hline Ta & $18 / 22(81.8 \%)$ \\
T1 & $4 / 22(18.2 \%)$ \\
\hline
\end{tabular}

Based on the EMT markers, we also demonstrated that $17.5 \%$ of NMIBC patients dis-

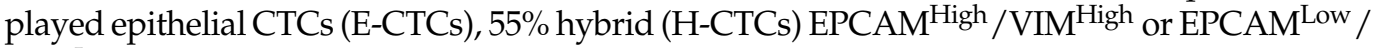
VIM $^{\text {Low }}$ and $27.5 \%$ displayed mesenchymal CTCs (M-CTCs) (Table 5). We then analyzed the distribution of patients, according to the T pathological stage (Tis, Ta and T1). We found that of 31 pTa patients, about $16 \%$ and $29 \%$ were E-CTCs or M-CTCs and 55\% were H-CTCs; of six pT1 patients, 33\% were E-CTCs and 67\% were H-CTCs; of three Tis patients, $33 \%$ were H-CTCs and $66 \%$ wee M-CTC (Table 5). 
Table 5. NMIBC patient distribution $(n=40)$ according to the different EMT phenotype and pathological T stage in blood CTCs.

\begin{tabular}{|c|c|c|c|}
\hline $\mathrm{T}$ & E-CTCs & H-CTCs & M-CTCs \\
\hline Tis $(n=3,7.5 \%)$ & $0(0.0 \%)$ & $1(33.3 \%)$ & $2(66.7 \%)$ \\
\hline Та $(n=31,77.5 \%)$ & $5(16.1 \%)$ & $17(54.8 \%)$ & $9(29.1 \%)$ \\
\hline $\mathrm{T} 1(n=6,15.0 \%)$ & $2(33.3 \%)$ & $4(66.7 \%)$ & $0(0.0 \%)$ \\
\hline
\end{tabular}

\subsection{Gene Expression Profile of the PD-L1 and EMT Genes in Blood CTCs from NMIBC Patients}

To evaluate the relation between the recurrence and the expression of PD-L1 and the EMT genes, the zinc finger E-box binding homebox 1 (ZEB1), Twist family BHLH transcription factor 1 (TWIST1), VIM and the invasive gene TIMP Metallopeptidase Inhibitor 2 (TIMP2) was evaluated. We stratified each marker expression patients in Low and High based on ROC analysis. PD-L1, TWIST1, VIM and TIMP2 expression levels showed a statistically significant difference in High respect Low-expression patients (Figure 3A). Moreover, a correlation index of studied genes confirmed the presence of a strong association between two different but related cluster genes: PD-L1 and TWIST1/TIMP2 and TIMP2 and ZEB1/VIM ( $p=0.05$ and 0.02 and $p=0.03$ and 0.05 , respectively) (Figure 3B). Thus, high PD-L1, TWIST1, VIM, ZEB1 and TIMP2 expression represents a specific gene signature in CTCs from NMIBC patients.

A
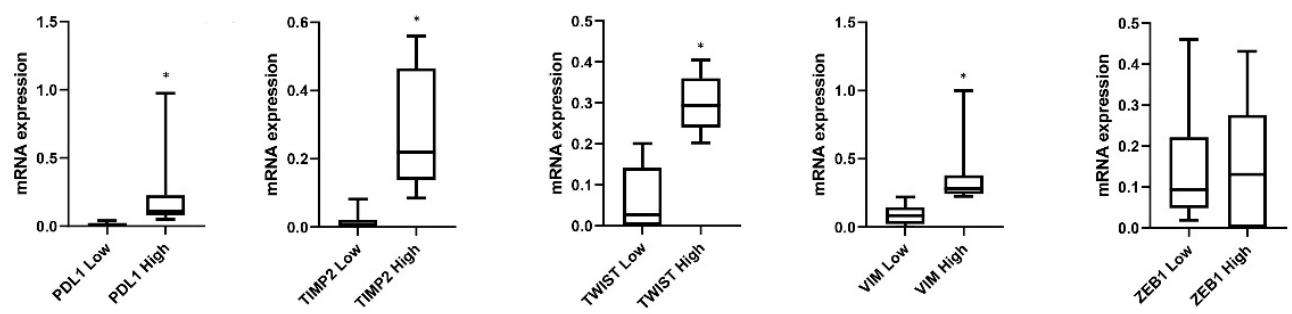

B

\begin{tabular}{lccc} 
Variable pairs & Coefficient of correlation & $\mathbf{P}$ \\
\hline PD-L1 - TIMP2 & 0.7182 & 0.05 \\
PD-L1 - TWIST1 & 0.8826 & 0.02 \\
\hline TIMP2 - ZEB1 & 0.8328 & 0.03 \\
TIMP2 - VIMENTIN & 0.7940 & 0.05
\end{tabular}

Figure 3. Expression of PD-L1, TIMP2, TWIST1, ZEB1 and VIM in CTCs isolated from NMIBC patients. (A) The expression was evaluated by ddPCR in NMIBC patients stratified according the recurrence at the first follow up. Expression values are presented as median with interquartile ranges (boxplot). $p$ value was based on the Mann-Whitney $U$ test, ${ }^{*} p<0.0001$. (B) Correlation index evaluated for PD-L1-TIMP2, PD-L1-TWIST1, TIMP2-ZEB1 and TIMP2-VIM variable pairs.

Furthermore, we analyzed the expression of PD-L1 mRNA in CTCs based on pathological pTa and pT1 stage (Figure 4). Higher PD-L1 expression was found in pT1 (22.5\%, $n=6 / 37)$ compared to pTa $(77.5 \%, n=31 / 37)$ NMIBC. Higher TIMP2 and VIM mRNA levels were found in pathological pT1 compared to pTa tumors in blood CTCs from NMIBC patients. No significant correlation was found for ZEB1 and TWIST1 markers. 

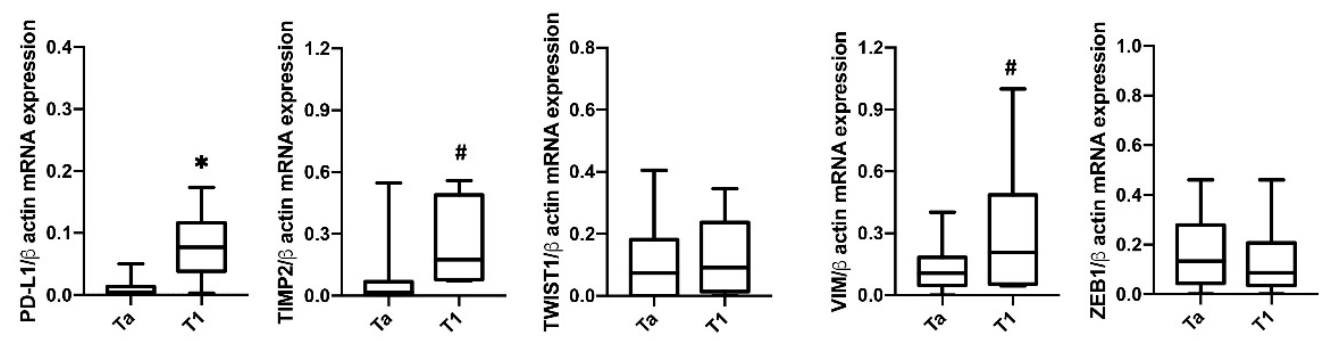

Figure 4. Expression of PD-L1, TIMP2, TWIST1, ZEB1 and VIM in CTCs isolated from NMIBC patients according the $\mathrm{T}$ stage. The expression was evaluated by ddPCR. Expression values are presented as median with interquartile ranges (boxplot). $p$ value was based on the Mann-Whitney $U$ test for PD-L1, TWIST1, ZEB1 and VIM. TIMP2 was analyzed by Welch's test. ${ }^{*} p<0.001,{ }^{\#} p<0.05$.

3.4. Correlation between PD-L1, EMT Genes and Recurrence-Free Survival (RFS) in CTCs from NMIBC Patients

Firstly, we scrutinized the correlation between clinicopathological features and RFS time to determine their prognostic significance by Kaplan-Meier (Table 6) and Univariate analyses (Table 7). No statistical significance was found among age, grade, $\mathrm{T}$ stage, sex and histology.

Table 6. Correlation analysis by Kaplan-Meier between patients' characteristics and RFS.

\begin{tabular}{|c|c|c|c|c|c|}
\hline Variables & $n$ & $p$-Value & HR & $95 \%$ CI & Chi-Square \\
\hline $\begin{array}{c}\text { Age: }<60 \text { vs. }>60 \text { years } \\
>60 \text { years }=29 / 40(72.5 \%) \text { vs. }<60 \text { years }=11 / 40(27.5 \%)\end{array}$ & 40 & $=0.2355$ & 0.4168 & $0.1545-1.5827$ & - \\
\hline $\begin{array}{l}\text { Pathological Grade: Low vs. High } \\
\text { Low Grade }=36 / 40(90 \%) \text { vs. High Grade }=4 / 40(10 \%)\end{array}$ & 40 & $=0.1719$ & 0.3344 & $0.1143-1.1234$ & - \\
\hline $\begin{array}{c}\text { T stage: Tis vs. Ta vs. T1 } \\
\text { Tis }=3 / 40(7.5 \%) \text { vs. Ta }=31 / 40(77.5 \%) \text { vs. } \mathrm{T} 1=6 / 40(15.0 \%)\end{array}$ & 40 & $=0.1019$ & & - & 4.5680 \\
\hline $\begin{array}{c}\text { Sex: Female vs. Male } \\
\text { Female }=7 / 40(17.5 \%) \text { vs. Male }=33 / 40(82.5 \%)\end{array}$ & 40 & $=0.6378$ & 0.6987 & $0.1879-2.7854$ & - \\
\hline $\begin{array}{c}\text { Histology: Papillary vs. Not Papillary } \\
\text { Papillary }=29 / 40 \text { (72.5\%) vs. Not Papillary }=11 / 40(27.5 \%)\end{array}$ & 40 & $=0.0832$ & 2.4631 & $0.8657-10.3978$ & - \\
\hline
\end{tabular}

Table 7. Univariate analysisof clinicopathological characteristics affecting RFS in NMIB patients.

\begin{tabular}{cccc}
\hline Variables & $n$ & $p$-Value & $\begin{array}{c}\text { Hazard Ratio } \\
\mathbf{( 9 5 \%} \mathbf{C I})\end{array}$ \\
\hline Age: $<60$ vs. $>60$ years & 40 & $=0.2594$ & $0.5335-10.5083$ \\
Pathological Grade: Low vs. High & 40 & $=0.8370$ & $0.2634-5.2006$ \\
T stage: Tis vs. Ta vs. T1 & 40 & $=0.1679$ & $0.8390-2.7830$ \\
Sex: Female vs. Male & 40 & $=0.6511$ & $0.3159-6.3487$ \\
Histology: Papillary vs. Not Papillary & 40 & $=0.0900$ & $0.1389-1.1474$ \\
\hline
\end{tabular}

In addition, the relation between RFS and PD-L1, VIM, TWIST1, ZEB1 and TIMP2 levels was carried out by Kaplan-Meier analysis (Figure 5). A significant correlation between high PD-L1, TWIST1, ZEB1 and TIMP2 and reduced RFS was evidenced. 

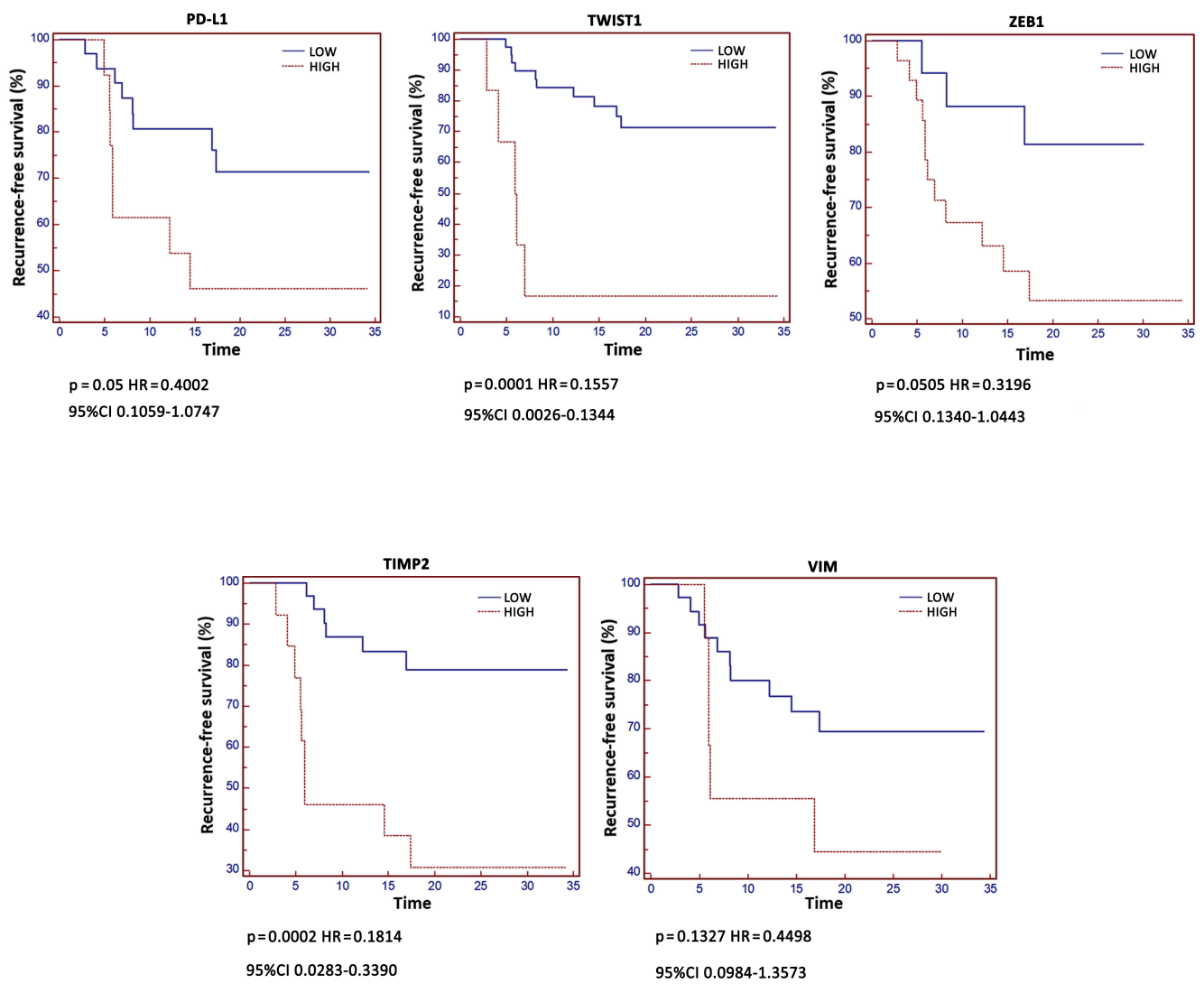

Figure 5. Kaplan-Meier analysis according to gene expression. Kaplan-Meier analysis was evaluated stratifying patients in High and Low expressing according to PD-L1, TIMP2, TWIST1, VIM and ZEB1 levels.

Univariate analysis confirmed that high PD-L1, TWIST1 and TIMP2 were significantly associated with shorter tumor recurrence ( $p$-value $<0.03,<0.02$ and $<0.05$, respectively). Moreover, by Multivariate analysis using Cox's proportional hazard model, high PD-L1 $(p<0.01)$, TWIST1 $(p<0.03)$, TIMP2 $(p=0.11)$ expression were independent and significant prognostic factors for tumor recurrence in NMIBC patients (Table 8).

Table 8. Univariate and multivariate Cox proportional hazards regression analysis for recurrent free survival (RFS) in NMIB patients.

\begin{tabular}{ccccc}
\hline \multirow{2}{*}{$\begin{array}{c}\text { Molecular } \\
\text { Variables }\end{array}$} & \multicolumn{2}{c}{ Univariate Analysis } & \multicolumn{2}{c}{ Multivariate Analysis } \\
\cline { 2 - 5 } & Hazard Ratio (95\% CI) & $\boldsymbol{p}$-Value & Hazard Ratio (95\% CI) & $\boldsymbol{p}$-Value \\
\hline PD-L1 & $1.0721-6.8003$ & $<0.03^{*}$ & $1.3267-9.5962$ & $<0.01^{*}$ \\
TWIST1 & $0.5286-3238.7085$ & $<0.05^{*}$ & $1.4868-8518.2648$ & $<0.03 *$ \\
TIMP2 & $1.5390-133.7713$ & $<0.02 *$ & $0.6190-128.9769$ & $=0.11$ \\
VIM & $0.2926-27.0342$ & $<0.33$ & $n s$ & $n s$ \\
ZEB1 & $0.1792-159.9790$ & $<0.36$ & $n s$ & $n s$ \\
\hline
\end{tabular}

$n s=$ non statistically significant; ${ }^{*} p<0.05$.

\section{Discussion}

Circulating tumor cells are usually assumed to be surrogates for micrometastatic disease. In BC, the risk of metastatic disease is clearly correlated to tumor stage. Indeed, the prognostically worse value of CTC has been demonstrated in MIBC and advanced urothelial BC [24,25]. CTCs represent an important circulating biomarker in NMIBC and were associated with disease recurrence and progression [26,27], although the mechanisms linking CTCs in the peripheral blood and local tumor recurrence in the bladder are still unknown. 
In this study, we demonstrated that $90 \%$ of NMIBC patients showed detectable CTCs, with a median CTC count of about four. By ScreenCell filtration method and ddPCR, as well as confocal microscopy analysis, the 93\% showed a canonical EpCAM+/CK+ CD45CTC phenotype. Higher PD-L1, TWIST1, VIM, ZEB1 and TIMP2 expression was evidenced in pT1 NMIBC patients, compared to pTa samples. Moreover, a strong association between PD-L1 and TWIST1 and TIMP2 and between TIMP2 and ZEB1 and VIM was evidenced by correlation index analysis.

Stratification of NMIBC patients into low and high PD-L1, TWIST1, VIM, ZEB1 and TIMP2 expression by ROC analysis permits the estimation of the risk of recurrence and progression, and it is also essential for decisions about adjuvant intravesical instillation [2]. Herein, by Kaplan-Meier analysis, we found that RFS was reduced in patients with high PD-L1 ( $p=0.05, \mathrm{HR}=0.4002,95 \% \mathrm{CI}=0.1059-1.0747)$ as well as TWIST1 $(p=0.0001, \mathrm{HR}=0.1557,95 \% \mathrm{CI}=0.0026-0.1344)$, ZEB1 $(p=0.0505, \mathrm{HR}=0.3196$, $95 \% \mathrm{CI}=0.1340-1.0443)$ and TIMP2 $(p=0.0002, \mathrm{HR}=0.1814,95 \% \mathrm{CI}=0.0283-0.3390)$ mRNA expression compared to those expressing low levels (6.5 vs. 17.4, 6.0 vs. 17.4, 6.9 vs. $17.4,5.5$ vs. 17.4 and 5.9 vs. 16.9 months, respectively). Similar results were obtained for PD-L1, TWIST1 and TIMP2 ( $p=0.03,0.02$ and 0.05, respectively), by univariate analysis. Finally, by multivariate analysis, high PD-L1 and TWIST1 expression was found to be independent and negative prognostic factors for tumor recurrence in NMIBC patients.

The prognostic significance of $P D-L 1$ in $B C$ has been associated with recurrence and poor survival [2]. However, data on $P D-L 1$ expression and its prognostic significance are controversial. Positive and high PD-L1 levels were found in 46\% and 7\% of NMIBC-HG, with the positive expression associated with submucosal invasion and refractory-tumor recurrence. Moreover, after BCG treatment, 55\% and 11\% of NMIBC-HG patients had positive and high PD-L1 levels [28]. Surprisingly, in T1-HG NMIBC patients, high PD-L1 mRNA expression represented a favorable prognostic factor for better RFS in the absence of anti-PD-1 or anti-PD-L1 treatments [29]. Thus, this high PD-L1 expression could be promising in the view of anti-PD-L1 therapies.

Similarly to our results, in ESCC, high PD-L1 tumor levels are associated with EMT and poor prognosis. High PD-L1 was associated with worse overall and relapse-free survival. Moreover, a correlation between PD-L1, ZEB1 expression and poor prognosis was found, suggesting that a cooperative mechanism bridging tumor immune avoidance and EMT contributes to tumor malignancy in ESCC [11].

In cancer cells, EMT-induced signals may be the results of EMT-transcription factors, including ZEB1 and TWIST1 [30]. The PD-L1 gene promoter region contains a ZEB1 binding site, which has led to speculation that $P D-L 1$ expression may be regulated by the ZEB1 transcription factor. TWIST1 and VIM correlate with recurrence in NMIBC patients [31,32]. Higher TIMP2 mRNA expression in superficial BC patients with recurrence, compared with those without recurrence, was found. In addition, Cox's multivariate analysis revealed that elevated TIMP2 was associated with a high incidence of intravesical recurrence [33]. Since CTCs are implicated in the metastatic spread, and in NMIBC they are associated with recurrence, it is intriguing that there is a high expression of invasive marker TIMP2 in recurrent NMIBC patients.

Overall, the high PD-L1 and EMT/invasive expression in blood CTCs from NMIBCs could identify patients with a super-high-risk of recurrence and progression. Moreover, it may change the treatment of super-high-risk NMIBC patients who have already a systemic disease at diagnosis and might therefore potentially benefit from systemic treatment with immune checkpoint inhibitors.

\section{Conclusions}

Our findings demonstrate the ability to detect CTCs in blood from NMIBC patients' and quantify the PD-L1, TWIST1, VIM, ZEB1 and TIMP2 mRNA expression using the ddPCR assay, showing high specificity and sensitivity. Exploration analysis of RFS suggests a trend toward worse recurrence time in those patients with high PD-L1, TWIST1, ZEB1 
and TIMP2 mRNA expression, strongly supporting the necessity to consider NMIBC patients as ideal candidates for systemic approaches with immune checkpoint inhibitors. While the data presented here are compelling, it should be underlined that the principal limitation of this study was the small sample size and requires validation with a larger study encompassing a broader patient population. Nonetheless, these data provide initial support for the broader development of PD-L1 and EMT CTC signature expression. With further study, the PD-L1/TWIST1/TIMP2 expression in CTCs isolated from peripheral blood could become new prognostic and predictive biomarkers to stratify treatment and to predict immunotherapy responses in low-grade NMIBCs.

Author Contributions: Conceptualization, G.S.; methodology, M.B.M. and C.A.; software, G.S.; validation, G.S., M.S., A.C. and R.M.; formal analysis, G.S.; investigation, M.B.M. and C.A.; resources, G.S. and R.M.; data curation, G.S.; writing—original draft preparation, G.S.; writing-review and editing, M.B.M., C.A., F.M., M.N., O.M., J.A.R.d.V., M.G., A.G., E.M., A.C. and R.M.; visualization, F.M.; supervision, G.S.; project administration, M.B.M. and G.S.; funding acquisition, G.S. Giorgio Santoni has full access to all the data in the study and takes responsibility for the integrity of the data and the accuracy of the data analysis. All authors have read and agreed to the published version of the manuscript.

Funding: This work was supported by grants from MIUR PRIN 2017 and Fondazione Umberto Veronesi (Post-doctoral Fellowship 2018, 2019 to M.B.M.).

Institutional Review Board Statement: The study was conducted according to the guidelines of the Declaration of Helsinki, and approved by the Ethical Committee CEAS UMBRIA (URO009-3171/18).

Informed Consent Statement: Informed consent was obtained from all subjects involved in the study. Written informed consent has been obtained from the patients to publish this paper.

Data Availability Statement: The data that support the findings of this study are available from the corresponding authors upon request.

Conflicts of Interest: The authors declare no conflict of interest.

\section{References}

1. Antoni, S.; Ferlay, J.; Soerjomataram, I.; Znaor, A.; Jemal, A.; Bray, F. Bladder Cancer Incidence and Mortality: A Global Overview and Recent Trends. Eur. Urol. 2017, 71, 96-108. [CrossRef]

2. Babjuk, M.; Böhle, A.; Burger, M.; Capoun, O.; Cohen, D.; Compérat, E.M.; Hernández, V.; Kaasinen, E.; Palou, J.; Rouprêt, M.; et al. EAU Guidelines on Non-Muscle-invasive Urothelial Carcinoma of the Bladder: Update 2016. Eur. Urol. 2017, 71, 447-461. [CrossRef] [PubMed]

3. Singer, S.; Ziegler, C.; Schwalenberg, T.; Hinz, A.; Götze, H.; Schulte, T. Quality of life in patients with muscle invasive and non-muscle invasive bladder cancer. Support. Care Cancer 2013, 21, 1383-1393. [CrossRef] [PubMed]

4. Witjes, J.A.; Bruins, H.M.; Cathomas, R.; Compérat, E.M.; Cowan, N.C.; Gakis, G.; Hernández, V.; Espinós, E.L.; Lorch, A.; Neuzillet, Y.; et al. European Association of Urology Guidelines on Muscle-invasive and Metastatic Bladder Cancer: Summary of the 2020 Guidelines. Eur. Urol. 2021, 79, 82-104. [CrossRef] [PubMed]

5. Xylinas, E.; Robinson, B.; Kluth, L.; Volkmer, B.; Hautmann, R.; Küfer, R.; Zerbib, M.; Kwon, E.; Thompson, R.; Boorjian, S.; et al. Association of T-cell co-regulatory protein expression with clinical outcomes following radical cystectomy for urothelial carcinoma of the bladder. Eur. J. Surg. Oncol. 2014, 40, 121-127. [CrossRef] [PubMed]

6. $\quad$ Powles, T.; Eder, J.P.; Fine, G.D.; Braiteh, F.S.; Loriot, Y.; Cruz, C.; Bellmunt, J.; Burris, H.A.; Petrylak, D.P.; Teng, S.-L.; et al. MPDL3280A (anti-PD-L1) treatment leads to clinical activity in metastatic bladder cancer. Nature 2014, 515, 558-562. [CrossRef] [PubMed]

7. Kawahara, T.; Ishiguro, Y.; Ohtake, S.; Kato, I.; Ito, Y.; Ito, H.; Makiyama, K.; Kondo, K.; Miyoshi, Y.; Yumura, Y.; et al. PD-1 and PD-L1 are more highly expressed in high-grade bladder cancer than in low-grade cases: PD-L1 might function as a mediator of stage progression in bladder cancer. BMC Urol. 2018, 18, 97. [CrossRef] [PubMed]

8. Balar, A.V.; Castellano, D.; O’Donnell, P.H.; Grivas, P.; Vuky, J.; Powles, T.; Plimack, E.R.; Hahn, N.M.; de Wit, R.; Pang, L.; et al. First-line pembrolizumab in cisplatin-ineligible patients with locally advanced and unresectable or metastatic urothelial cancer (KEYNOTE-052): A multicentre, single-arm, phase 2 study. Lancet Oncol. 2017, 18, 1483-1492. [CrossRef]

9. Rosenberg, J.E.; Hoffman-Censits, J.; Powles, T.; van der Heijden, M.S.; Balar, A.V.; Necchi, A.; Dawson, N.; O’Donnell, P.H.; Balmanoukian, A.; Loriot, Y.; et al. Atezolizumab in patients with locally advanced and metastatic urothelial carcinoma who have progressed following treatment with platinum-based chemotherapy: A single-arm, multicentre, phase 2 trial. Lancet 2016, 387, 1909-1920. [CrossRef] 
10. Mahmoudian, R.A.; Mozhgani, S.; Abbaszadegan, M.R.; Mokhlessi, L.; Montazer, M.; Gholamin, M. Correlation between the immune checkpoints and EMT genes proposes potential prognostic and therapeutic targets in ESCC. J. Mol. Histol. 2021, 52, 597-609. [CrossRef]

11. Tsutsumi, S.; Saeki, H.; Nakashima, Y.; Ito, S.; Oki, E.; Morita, M.; Oda, Y.; Okano, S.; Maehara, Y. Programmed death-ligand 1 expression at tumor invasive front is associated with epithelial-mesenchymal transition and poor prognosis in esophageal squamous cell carcinoma. Cancer Sci. 2017, 108, 1119-1127. [CrossRef]

12. Lamouille, S.; Xu, J.; Derynck, R. Molecular mechanisms of epithelial-mesenchymal transition. Nat. Rev. Mol. Cell Biol. 2014, 15, 178-196. [CrossRef] [PubMed]

13. Na, L.; Wang, Z.; Bai, Y.; Sun, Y.; Dong, D.; Wang, W.; Zhao, C. WNT7B represses epithelial-mesenchymal transition and stem-like properties in bladder urothelial carcinoma. Biochim. Biophys. Acta Mol. Basis Dis. 2022, 1868, 166271. [CrossRef]

14. Wang, H.; Mei, Y.; Luo, C.; Huang, Q.; Wang, Z.; Lu, G.-M.; Qin, L.; Sun, Z.; Huang, C.-W.; Yang, Z.-W.; et al. Single-Cell Analyses Reveal Mechanisms of Cancer Stem Cell Maintenance and Epithelial-Mesenchymal Transition in Recurrent Bladder Cancer. Clin. Cancer Res. 2021, 27, 6265-6278. [CrossRef] [PubMed]

15. Breyer, J.; Gierth, M.; Shalekenov, S.; Aziz, A.; Schäfer, J.; Burger, M.; Denzinger, S.; Hofstädter, F.; Giedl, C.; Otto, W. Epithelialmesenchymal transformation markers E-cadherin and survivin predict progression of stage pTa urothelial bladder carcinoma. World J. Urol. 2016, 34, 709-716. [CrossRef]

16. Alsuliman, A.; Colak, D.; Al-Harazi, O.; Fitwi, H.; Tulbah, A.; Al-Tweigeri, T.; Al-Alwan, M.; Ghebeh, H. Bidirectional crosstalk between PD-L1 expression and epithelial to mesenchymal transition: Significance in claudin-low breast cancer cells. Mol. Cancer 2015, 14, 149. [CrossRef] [PubMed]

17. Ock, C.-Y.; Kim, S.; Keam, B.; Kim, M.; Kim, T.M.; Kim, J.-H.; Jeon, Y.K.; Lee, J.-S.; Kwon, S.K.; Hah, J.H.; et al. PD-L1 expression is associated with epithelial-mesenchymal transition in head and neck squamous cell carcinoma. Oncotarget 2016, 7, 15901-15914. [CrossRef]

18. Santoni, G.; Morelli, M.B.; Amantini, C.; Battelli, N. Urinary Markers in Bladder Cancer: An Update. Front. Oncol. 2018, 8, 362. [CrossRef]

19. Chalfin, H.J.; Kates, M.; van der Toom, E.E.; Glavaris, S.; Verdone, J.E.; Hahn, N.M.; Pienta, K.J.; Bivalacqua, T.J.; Gorin, M.A. Characterization of Urothelial Cancer Circulating Tumor Cells with a Novel Selection-Free Method. Urology 2018, 115, 82-86. [CrossRef]

20. Massagué, J.; Obenauf, A.C. Metastatic colonization by circulating tumour cells. Nature 2016, 529, 298-306. [CrossRef]

21. Jiang, H.; Gu, X.; Zuo, Z.; Tian, G.; Liu, J. Prognostic value of circulating tumor cells in patients with bladder cancer: A meta-analysis. PLoS ONE 2021, 16, e0254433. [CrossRef] [PubMed]

22. Nicolazzo, C.; Colangelo, L.; Corsi, A.; Carpino, G.; Gradilone, A.; Sonato, C.; Raimondi, C.; Gaudio, E.; Gazzaniga, P.; Gianni, W. Liquid Biopsy in Rare Cancers: Lessons from Hemangiopericytoma. Anal. Cell. Pathol. 2018, 2018, 9718585. [CrossRef]

23. Msaouel, P.; Koutsilieris, M. Diagnostic value of circulating tumor cell detection in bladder and urothelial cancer: Systematic review and meta-analysis. BMC Cancer 2011, 11, 336. [CrossRef] [PubMed]

24. Soave, A.; Riethdorf, S.; Dahlem, R.; Von Amsberg, G.; Minner, S.; Weisbach, L.; Engel, O.; Fisch, M.; Pantel, K.; Rink, M. A nonrandomized, prospective, clinical study on the impact of circulating tumor cells on outcomes of urothelial carcinoma of the bladder patients treated with radical cystectomy with or without adjuvant chemotherapy. Int. J. Cancer 2017, 140, 381-389. [CrossRef] [PubMed]

25. Anantharaman, A.; Friedlander, T.; Lu, D.; Krupa, R.; Premasekharan, G.; Hough, J.; Edwards, M.; Paz, R.; Lindquist, K.; Graf, R.; et al. Programmed death-ligand 1 (PD-L1) characterization of circulating tumor cells (CTCs) in muscle invasive and metastatic bladder cancer patients. BMC Cancer 2016, 16, 744. [CrossRef] [PubMed]

26. Gazzaniga, P.; De Berardinis, E.; Raimondi, C.; Gradilone, A.; Busetto, G.M.; De Falco, E.; Nicolazzo, C.; Giovannone, R.; Gentile, V.; Cortesi, E.; et al. Circulating tumor cells detection has independent prognostic impact in high-risk non-muscle invasive bladder cancer. Int. J. Cancer 2014, 135, 1978-1982. [CrossRef]

27. Busetto, G.M.; Ferro, M.; Del Giudice, F.; Antonini, G.; Chung, B.I.; Sperduti, I.; Giannarelli, D.; Lucarelli, G.; Borghesi, M.; Musi, G.; et al. The Prognostic Role of Circulating Tumor Cells (CTC) in High-risk Non-muscle-invasive Bladder Cancer. Clin. Genitourin. Cancer 2017, 15, e661-e666. [CrossRef]

28. Aydin, A.M.; Baydar, D.E.; Hazir, B.; Babaoglu, B.; Bilen, C.Y. Prognostic significance of pre- and post-treatment PD-L1 expression in patients with primary high-grade non-muscle-invasive bladder cancer treated with BCG immunotherapy. World J. Urol. 2020, 38, 2537-2545. [CrossRef]

29. Breyer, J.; Wirtz, R.M.; Otto, W.; Erben, P.; Worst, T.; Stoehr, R.; Eckstein, M.; Denzinger, S.; Burger, M.; Hartmann, A. High PDL1 mRNA expression predicts better survival of stage pT1 non-muscle-invasive bladder cancer (NMIBC) patients. Cancer Immunol. Immunother. 2018, 67, 403-412. [CrossRef]

30. Jang, M.H.; Kim, H.J.; Kim, E.J.; Chung, Y.R.; Park, S.Y. Expression of epithelial-mesenchymal transition-related markers in triple-negative breast cancer: ZEB1 as a potential biomarker for poor clinical outcome. Hum. Pathol. 2015, 46, 1267-1274. [CrossRef]

31. Zhao, D.; Besser, A.H.; Wander, S.A.; Sun, J.; Zhou, W.; Wang, B.; Ince, T.; Durante, M.; Guo, W.; Mills, G.; et al. Cytoplasmic p27 promotes epithelial-mesenchymal transition and tumor metastasis via STAT3-mediated Twist1 upregulation. Oncogene 2015, 34, 5447-5459. [CrossRef] [PubMed] 
32. Liu, B.; Miyake, H.; Nishikawa, M.; Fujisawa, M. Expression profile of epithelial-mesenchymal transition markers in non-muscleinvasive urothelial carcinoma of the bladder: Correlation with intravesical recurrence following transurethral resection. Urol. Oncol. Semin. Orig. Investig. 2014, 33, 110.e11-110.e18. [CrossRef] [PubMed]

33. Hara, I.; Miyake, H.; Hara, S.; Arakawa, S.; Kamidono, S. Significance of Matrix Metalloproteinases and Tissue Inhibitors of Metalloproteinase Expression in the Recurrence of Superficial Transitional Cell Carcinoma of the Bladder. J. Urol. 2001, 165, 1769-1772. [CrossRef] 